\title{
COVID-19 Interpretable Diagnosis Algorithm Based on a Small Number of Chest X-Ray Samples
}

\author{
$B U \operatorname{Ran}($ 卜 由), XIANG Wei* (向 伟), CAO Shitong (曹世同) \\ (Key Laboratory of Electronic and Information Engineering, \\ State Ethnic Affairs Commission (Southwest Minzu University), Chengdu 610041, China)
}

(C) Shanghai Jiao Tong University and Springer-Verlag GmbH Germany, part of Springer Nature 2021

\begin{abstract}
The COVID-19 medical diagnosis method based on individual's chest X-ray (CXR) is achieved difficultly in the initial research, owing to difficulties in identifying CXR data of COVID-19 individuals. At the beginning of the study, infected individuals' CXRs were scarce. The combination of artificial intelligence (AI) and medical diagnosis has been advanced and popular. To solve the difficulties, the interpretability analysis of AI model was used to explore the pathological characteristics of CXR samples infected with COVID-19 and assist in medical diagnosis. The dataset was expanded by data augmentation to avoid overfitting. Transfer learning was used to test different pre-trained models and the unique output layers were designed to complete the model training with few samples. In this study, the output results of four pre-trained models in three different output layers were compared, and the results after data augmentation were compared with the results of the original dataset. The control variable method was used to conduct independent tests of 24 groups. Finally, 99.23\% accuracy and $98 \%$ recall rate were obtained, and the visual results of CXR interpretability analysis were displayed. The network of COVID-19 interpretable diagnosis algorithm has the characteristics of high generalization and lightweight. It can be quickly applied to other urgent tasks with insufficient experimental data. At the same time, interpretability analysis brings new possibilities for medical diagnosis.
\end{abstract}

Key words: COVID-19, chest X-ray (CXR), interpretability, data augmentation, transfer learning, convolutional neural network

CLC number: TP 183, R 445 Document code: A

\section{Introduction}

Research data of COVID-19 breaking out at the end of 2019 were scarce for a long time. And because of the rapid spread of COVID-19 and the special pathological features that are difficult to identify, there is a serious lack of chest X-ray (CXR) data of COVID-19 patients that can be used for scientific research. Pneumonia is very common in clinic, but accurate diagnosis of pneumonia is a difficult task. Diagnosis of pneumonia requires examination of CXR samples by medical experts and confirmation by clinical history, vital signs and medical laboratory tests. Pneumonia usually presents as an area of increased opacity on the CXR, but there are many other lung diseases that interfere

Received: 2021-04-12 Accepted: 2021-04-21

Foundation item: the Southwest Minzu University Graduate Innovative Research Project (No. CX2020SZ95, Master Program); the National Natural Science Foundation of China (No. 62073270); the State Ethnic Affairs Commission Innovation Research Team Project, and Innovative Research Team Project of the Education Department of Sichuan Province (No. 15TD0050)

*E-mail: 21500068@swun.edu.cn with the diagnosis of pneumonia, such as pulmonary edema, bleeding, volume reduction (atelectasis or collapse), lung cancer, and lung changes after radiotherapy or surgery. In addition, the pleural fluid (pleural effusion) outside the lung also shows increased opacity on the CXR. CXR analysis requires radiologists to spend a lot of time, but the pathological data of COVID-19 CXR data are not obvious. Doctors and medical resources are very scarce all over the world at the time of the disaster ${ }^{[1]}$. Therefore, it is of practical significance to develop a targeted intelligent analysis system to save the precious time of medical professionals.

With the support of massive data, the combination of artificial intelligence (AI) and medical science develops rapidly and efficiently. Compared with conventional detection methods such as nucleic acid detection, $\mathrm{AI}$ algorithm detection to avoid the risk of secondary infection is more efficient and affordable. However, the lack of research data caused by the sudden COVID19 makes AI difficult to play its efficient and accurate advantage in this epidemic. To deal with such emergencies, we need to explore how to apply AI methods in the case of a small number of samples. The conventional 
deep learning models and methods are not suitable for the emergencies like COVID-19. The requirements of a few data, fast training fitting and convenient application have become the most important standards for evaluation models. Therefore, it is more practical to design a model with high precision, lightweight and easy implement.

AI application is a "black box" in many occasions. After training with massive data, the network can make judgments according to the characteristics that human beings cannot understand. Compared with computed tomography (CT) as a diagnostic source ${ }^{[2-3]}$, CXR diagnosis has a main obstacle to the visual confirmation of COVID-19 details to diagnose whether an individual is infected. It is difficult for doctors to judge the symptoms of COVID-19 in CXR and make diagnosis by CXR. For this study of COVID-19, we not only use AI model to determine whether individuals are infected ${ }^{[4]}$, but also explore the criterion of AI system to make diagnosis. The interpretability study helps doctors to judge whether an individual is infected with COVID-19. An "AI doctor" is designed to judge whether there is an area infected with COVID-19 in a CXR. If there is a suspected infection area, the system will automatically mark the area to assist doctors in diagnosis, and the system will automatically mark one or several areas according to the weights to assist in medical diagnosis ${ }^{[5]}$. The addition of interpretability is not only to make individuals trust the trained model by understanding the criterion of AI model for judgment; it is more significant that the AI system analysis methods must be different from human beings. It would be a great contribution if the representative pathological features of COVID-19 could be found in the suspected infection areas marked by our AI model ${ }^{[6]}$.

The remarkable achievement of AI in medical science is based on the support of massive data; however, the COVID-19 is unexpected, new and unknown. For months, only a few hundred authoritative medical image data are available for scientific research, which puts forward new requirements for scientific research. VGG16 network is chosen as the backbone of the network because of its lightweight structure and remarkable effect. Complex networks with deeper structure may have better effect, but they are not suitable for the current situation of emergency. VGG-16 network is our best choice after many experiments and comparisons, but the network structure still needs to be improved, and a few data are still not enough to train a complete VGG-16 network. Therefore, the idea of transfer learning is applied, and VGG-16 network with pre-trained weights on ImageNet is used, and its original full connection layer is improved. The weight parameters of VGG-16 convolution layers are frozen, and the network full connection layers are improved to the structure of Average Pooling layer + Dense layer (ReLU activation function $)+$ Batch Normalization + Dropout + Dense layer (softmax activation function). After many comparative experiments, the redesigned network only needs a few training data and achieves good results. The improved network only needs 25 epochs training to converge, and much less training time in comparison to the ordinary deep learning model. Owing to the lightweight network structure and low-cost training process, the improved AI model can be more widely used in reality. In the case of only using CPU, a series of work can be completed within $10 \mathrm{~min}$, which is more meaningful contribution to underdeveloped areas.

In terms of medical level and infrastructure, almost all hospitals are equipped with X-ray imagers. Therefore, this design does not need other special test kits, and only uses CXR data to test COVID-19. Compared with CT scanning, CXR is easier to get, especially in rural areas and remote areas. CXR diagnosis can not only contribute to their health and epidemic prevention work, but also find more potential data, thus forming a positive cycle to solve COVID-19 as soon as possible. In addition, CXR has great advantages in popularity and cost compared with CT scanning.

\section{Relevant Work}

\subsection{COVID-19 and VGGNet}

The main clinical manifestations of COVID-19 were fever, dry cough and fatigue. Pulmonary imaging examination is of great value in the diagnosis, efficacy monitoring and discharge evaluation of COVID-19. AI technology perfectly matches the epidemic diagnosis task. The most important contribution of the combination of AI and medical science is to reduce the repeated work of doctors. Appropriate networks can distinguish normal individual and infected individual with high accuracy by CXR data.

VGGNet, a deep convolutional neural network, explores the relationship between the depth of convolution neural network and its performance. By repeatedly stacking $3 \times 3$ convolution kernel and $2 \times 2$ maximum pooling layer, a convolution neural network with 16-19 layers is constructed. VGGNet won the second place in the ILSVRC 2014 competition and won the title in positioning projects. Until now, VGGNet is still very suitable to be used to extract image features.

VGGNet uses $3 \times 3$ convolution core and $2 \times 2$ pooling core to improve the performance by deepening the network structure. The increase of network layers will not lead to the explosion of parameters, because the parameters are mainly concentrated in the last three fully connected layers. The series connection of two $3 \times 3$ convolution layers is equivalent to a $5 \times 5$ convolution layer, and the series connection of three $3 \times 3$ convolution layers is equivalent to a $7 \times 7$ convolution layer. 
In other words, the receptive field of three $3 \times 3$ convolution layers is equivalent to a $7 \times 7$ convolution layer. However, the parameters of three $3 \times 3$ convolution layers are only about half of $7 \times 7$, and the $3 \times 3$ combination has three nonlinear operations, which makes the former structure more capable of learning features ${ }^{[7]}$. VGGNet is often used in feature extraction and transfer learning tasks. The most widely used models are VGG-16 and VGG-19, which represent 16 and 19 layers of network, respectively. This is also the reason why VGG-16 and VGG-19 are selected as the test backbones. The VGGNet structure is shown in Fig. 1.

\begin{tabular}{|c|c|c|c|c|c|}
\hline \multicolumn{6}{|c|}{ ConvNet configuration } \\
\hline$\overline{\mathrm{A}}$ & A-LRN & $\mathrm{B}$ & $\mathrm{C}$ & $\mathrm{D}$ & $\mathrm{E}$ \\
\hline $\begin{array}{c}11 \text { weight } \\
\text { layers }\end{array}$ & $\begin{array}{c}11 \text { weight } \\
\text { layers }\end{array}$ & $\begin{array}{c}13 \text { weight } \\
\text { layers }\end{array}$ & $\begin{array}{c}16 \text { weight } \\
\text { layers }\end{array}$ & $\begin{array}{c}16 \text { weight } \\
\text { layers }\end{array}$ & $\begin{array}{c}19 \text { weight } \\
\text { layers }\end{array}$ \\
\hline \multicolumn{6}{|c|}{ Input $(224 \times 224$ RGB image $)$} \\
\hline Conv3-64 & $\begin{array}{c}\text { Conv3-64 } \\
\text { LRN }\end{array}$ & $\begin{array}{l}\text { Conv3-64 } \\
\text { Conv3-64 }\end{array}$ & $\begin{array}{l}\text { Conv3-64 } \\
\text { Conv3-64 }\end{array}$ & $\begin{array}{l}\text { Conv3-64 } \\
\text { Conv3-64 }\end{array}$ & $\begin{array}{l}\text { Conv3-64 } \\
\text { Conv3-64 }\end{array}$ \\
\hline \multicolumn{6}{|c|}{ maxpool } \\
\hline Conv3-128 & Conv3-128 & $\begin{array}{l}\text { Conv3-128 } \\
\text { Conv3-128 }\end{array}$ & $\begin{array}{l}\text { Conv3-128 } \\
\text { Conv3-128 }\end{array}$ & $\begin{array}{l}\text { Conv3-128 } \\
\text { Conv3-128 }\end{array}$ & $\begin{array}{l}\text { Conv3-128 } \\
\text { Conv3-128 }\end{array}$ \\
\hline \multicolumn{6}{|c|}{ Maxpool } \\
\hline $\begin{array}{l}\text { Conv3-256 } \\
\text { Conv3-256 }\end{array}$ & $\begin{array}{l}\text { Conv3-256 } \\
\text { Conv3-256 }\end{array}$ & $\begin{array}{l}\text { Conv3-256 } \\
\text { Conv3-256 }\end{array}$ & $\begin{array}{l}\text { Conv3-256 } \\
\text { Conv3-256 } \\
\text { Conv1-256 }\end{array}$ & $\begin{array}{l}\text { Conv3-256 } \\
\text { Conv3-256 } \\
\text { Conv3-256 }\end{array}$ & $\begin{array}{l}\text { Conv3-256 } \\
\text { Conv3-256 } \\
\text { Conv3-256 } \\
\text { Conv3-256 }\end{array}$ \\
\hline \multicolumn{6}{|c|}{ Maxpool } \\
\hline $\begin{array}{l}\text { Conv3-512 } \\
\text { Conv3-512 }\end{array}$ & $\begin{array}{l}\text { Conv3-512 } \\
\text { Conv3-512 }\end{array}$ & $\begin{array}{l}\text { Conv3-512 } \\
\text { Conv3-512 }\end{array}$ & $\begin{array}{l}\text { Conv3-512 } \\
\text { Conv3-512 } \\
\text { Conv1-512 }\end{array}$ & $\begin{array}{l}\text { Conv3-512 } \\
\text { Conv3-512 } \\
\text { Conv3-512 }\end{array}$ & $\begin{array}{l}\text { Conv3-512 } \\
\text { Conv3-512 } \\
\text { Conv3-512 } \\
\text { Conv3-512 }\end{array}$ \\
\hline \multicolumn{6}{|c|}{ Maxpool } \\
\hline $\begin{array}{l}\text { Conv3-512 } \\
\text { Conv3-512 }\end{array}$ & $\begin{array}{l}\text { Conv3-512 } \\
\text { Conv3-512 }\end{array}$ & $\begin{array}{l}\text { Conv3-512 } \\
\text { Conv3-512 }\end{array}$ & $\begin{array}{l}\text { Conv3-512 } \\
\text { Conv3-512 } \\
\text { Conv1-512 }\end{array}$ & $\begin{array}{l}\text { Conv3-512 } \\
\text { Conv3-512 } \\
\text { Conv3-512 }\end{array}$ & $\begin{array}{l}\text { Conv3-512 } \\
\text { Conv3-512 } \\
\text { Conv3-512 } \\
\text { Conv3-512 }\end{array}$ \\
\hline \multicolumn{6}{|c|}{ Maxpool } \\
\hline \multicolumn{6}{|c|}{ FC-4096 } \\
\hline \multicolumn{6}{|c|}{ FC-4096 } \\
\hline \multicolumn{6}{|c|}{ FC-1000 } \\
\hline \multicolumn{6}{|c|}{ Softmax } \\
\hline
\end{tabular}

Fig. 1 Different VGGNet configurations

In general, VGGNet has no advantage in accuracy compared with some recently proposed networks, e.g., Google's idea of inception structure, and ResNet whose depth and accuracy are greatly improved ${ }^{[8]}$. However, the results of these complex networks are obtained after the training of massive and complete training dataset. In practical application, how to build the most suitable and convenient network needs to pass the experimental tests.

\subsection{Data Augmentation}

A large number of samples are needed to train the model, so that the model can learn the sample features as much as possible. How to use AI method to solve the problem in the case of a small number of samples has become the difficulty of the design. In the initial study, too few training CXR data were obtained for experiments. In the case of no more reliable data, we use lightweight pre-trained model and data augmentation method to expand the dataset. More importantly, in the case of insufficient data, the training model is more practical and more suitable for emergencies.

The neural networks are not "smart" when the machine starts training. However, just make minor changes to the existing dataset. For example, when the picture is flipped, shifted, and rotated at different angles, or the brightness of the image is changed, the network is then equivalent to obtaining different data samples ${ }^{[9]}$. Figure 2 shows the effects of image flipping and rotation at different angles in the data augmentation method.

The reason why convolutional neural network can use data augmentation method is that it has translation invariance. If the target is placed in different conditions, additional data can be generated to train the neural 


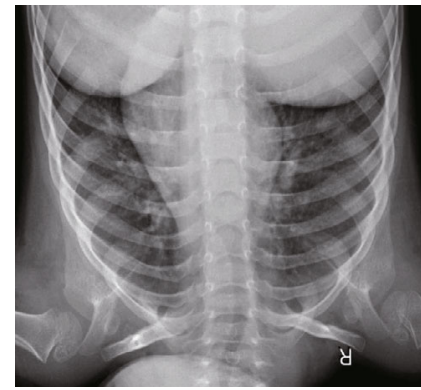

(a) Flipped image

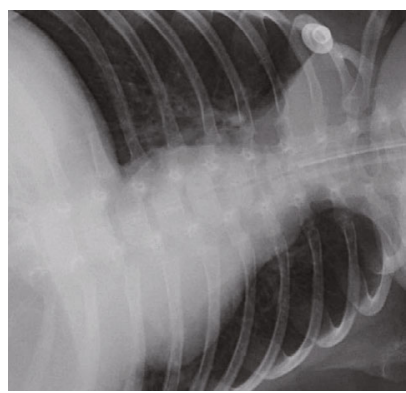

(b) Rotated image
Fig. 2 Image flipping and rotation at different angles

network. This is also in line with the human visual experience. It also needs to observe the target from different angles, so as to get a deeper feeling.

\section{Improved COVID-19 Network Based on VGG-16}

\subsection{Pre-trained Model}

Neural networks need a lot of data for training to get information from the data and convert it into corresponding weight. Obtained weights can be extracted and transferred to other neural networks. Transfer learning can reduce the cost of training AI models and make us avoid training a network from scratch. In the case of insufficient data, the pre-trained model learned by transfer learning may help train a network quickly ${ }^{[10]}$.

In subsequent experiments, VGG-16 network, VGG19 network, InceptionV3 network, ResNet50 network and Xception network are separately used as the backbone of the design. In addition, the weights in the convolutional layers of the pre-trained model were frozen, and full connection layers trained on different datasets were tested.

\subsection{Improved Version of the Full Connection Layer}

The purpose of this study was to determine whether COVID-19 symptoms were present in CXR images, and this is a binary classification. However, the traditional VGG-16 network deals with the problem of 1000 classification. Thus, the last three layers of full connection layer in the traditional VGG-16 network are abandoned. To solve this problem, an improved full connection layer was designed ${ }^{[11-13]}$.

The weight parameters of the convolutional layer after "ImageNet" pre-trained were firstly loaded and frozen so that it was not updated during training. And then, "Average Pooling" was used. This approach avoids overfitting in this layer and it can sum and average spatial information, and it is more robust to spatial variation of input. In addition, for the purpose of full connection preparation of "dense", "flatten" operation is used. Meanwhile, "dropout" is added between the full connection layers, so as to adjust the hyperparameter and avoid overfitting. What is noticeable is that the results with or without the "batch normalization (BN)" operation are also compared. Adding the BN layer can improve the gradient through the network and allow for greater learning rates and dramatically increase the training speed. Lastly, a dense layer was used to achieve a binary classification task. Table 1 shows the specific network structure. During the work, $80 \%$ of the CXR data were used for training and $20 \%$ of the CXR data for testing .

Table 1 Network structure

\begin{tabular}{|c|c|c|c|c|}
\hline $\begin{array}{l}\text { Layer } \\
\text { type }\end{array}$ & $\begin{array}{c}\text { Kernel } \\
\text { size }\end{array}$ & Stride & Output & $\begin{array}{c}\text { Activation } \\
\text { function }\end{array}$ \\
\hline Conv3-64 & $3 \times 3$ & 1 & $224 \times 224 \times 64$ & ReLU \\
\hline Conv3-64 & $3 \times 3$ & 1 & $224 \times 224 \times 64$ & ReLU \\
\hline Maxpool & $2 \times 2$ & 2 & $112 \times 112 \times 64$ & \\
\hline Conv3-128 & $3 \times 3$ & 1 & $112 \times 112 \times 128$ & ReLU \\
\hline Conv3-128 & $3 \times 3$ & 1 & $112 \times 112 \times 128$ & ReLU \\
\hline Maxpool & $2 \times 2$ & 2 & $56 \times 56 \times 128$ & \\
\hline Conv3-256 & $3 \times 3$ & 1 & $56 \times 56 \times 256$ & ReLU \\
\hline Conv3-256 & $3 \times 3$ & 1 & $56 \times 56 \times 256$ & ReLU \\
\hline Conv3-256 & $3 \times 3$ & 1 & $56 \times 56 \times 256$ & ReLU \\
\hline Maxpool & $2 \times 2$ & 2 & $28 \times 28 \times 256$ & \\
\hline Conv3-512 & $3 \times 3$ & 1 & $28 \times 28 \times 512$ & ReLU \\
\hline Conv3-512 & $3 \times 3$ & 1 & $28 \times 28 \times 512$ & ReLU \\
\hline Conv3-512 & $3 \times 3$ & 1 & $28 \times 28 \times 512$ & ReLU \\
\hline Maxpool & $2 \times 2$ & 2 & $14 \times 14 \times 512$ & \\
\hline Conv3-512 & $3 \times 3$ & 1 & $14 \times 14 \times 512$ & ReLU \\
\hline Conv3-512 & $3 \times 3$ & 1 & $14 \times 14 \times 512$ & ReLU \\
\hline Conv3-512 & $3 \times 3$ & 1 & $14 \times 14 \times 512$ & ReLU \\
\hline Averagepool & $2 \times 2$ & 2 & $7 \times 7 \times 512$ & \\
\hline Dense & & & $1 \times 1 \times 64$ & ReLU \\
\hline $\mathrm{BN}$ & & & $1 \times 1 \times 64$ & \\
\hline Dropout & & & $1 \times 1 \times 64$ & \\
\hline Dense & & & $1 \times 1 \times 2$ & Softmax \\
\hline
\end{tabular}

\subsection{Interpretability and Improvement Strate-} gies

AI is an "in-out" structure, and on many occasions networks trained with massive data make judgments based on features that are difficult for humans to understand ${ }^{[14]}$. One of the interpretability tools, "Lime", interprets the model in an understandable way. Meanwhile, it takes into account human habits and the explanation is not too long.

Here, an example of image recognition is given to explain how "Lime" makes explanation about judgments. For image input, assume that you want to explain the classification criterion of a classifier. As shown in Fig. 3, the classifier is predictive of the likelihood that an image contains a tree frog. 


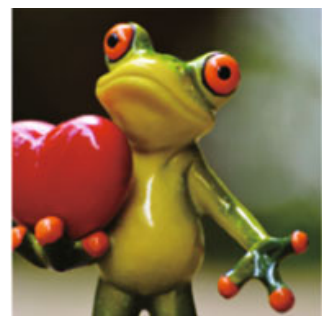

(a) Original

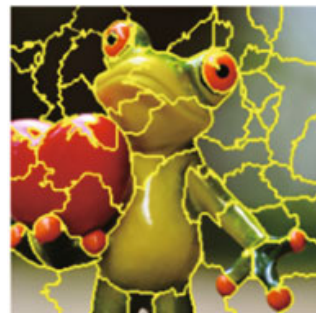

(b) Decomposed
Fig. 3 Original and decomposed images

First, we divide the image on the left into interpretable components based on successive pixels. A disturbed dataset is generated by "masking" part of the images (in this case, the masked region turns gray). Second, we reenter each modified instance into the model and obtain the accuracy of the output of the disturbed image. We train a linear model on this disturbed dataset containing contribution weights of all pixel blocks. We are more concerned about the regions in disturbed images that have a greater impact on the model judgment. Finally, we tend to take several pixel blocks with the highest weight as an example to explain and display everything else gray.

The contribution to the model judgment by calculating the pixel blocks except the gray area is shown in Fig. 4. After locally weighted regression, the regions with the greatest contribution are marked in the image.

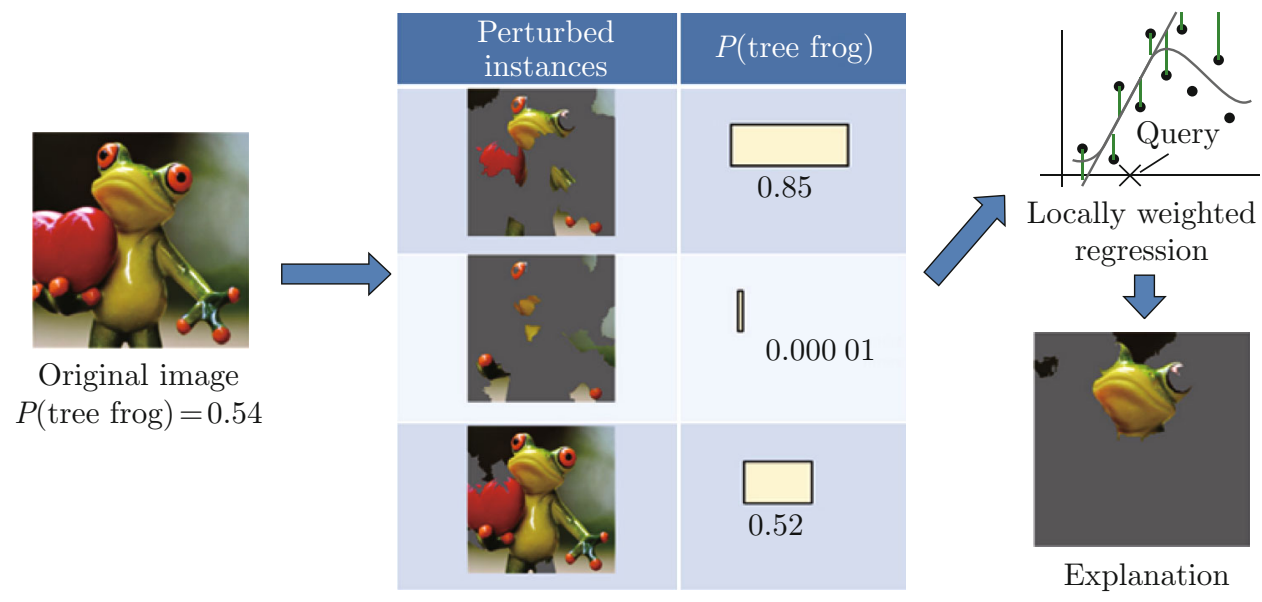

Fig. 4 Calculation of the contribution of each region

To explore the diagnostic criterion of the model rather than just focusing on accuracy, we add the interpretable method "Lime" to the network when tested on the COVID-19 dataset. Then, CXR data are analyzed, the suspected infection areas are identified and they are distinguished with different colors to better explain and provide more information. Green represents the areas that contribute to the judgment. The red is the area that impedes judgment.

First, the input CXR image is divided into interpretable blocks based on contiguous pixels. A disturbed dataset is generated by randomly "closing" some blocks in CXR. If the predicted score of the disturbed CXR is significantly different from that of the original CXR, it proves that the disturbed blocks play an important role in the process of model judgment. Each disturbed sample is re-input into the model to obtain a new output diagnostic probability. Then, a linear model is trained on the disturbed dataset, and it can be found that the blocks have a greater impact on the results. Finally, the pixel blocks with the highest weight are taken as the explanation. In other words, small perturbations are made around the inputs and the results of the model are recorded to figure out which input parts contribut- ing more to the predicted results. The weights are assigned according to the distance from the data points of these disturbances to the original data. Based on above learning, an explicable prediction is got.

However, "Lime" also has shortcomings. It has a loophole for medical target detection because the "Lime" interpreter's dividing and regrouping operation is completely random. There is a possibility of missing the important parts of the whole CXR's block combination. For COVID-19 detection interpretation studies, the interpretation area should focus upon lungs rather than the entire chest cavity. In the interpretable analysis, the features of the input CXR image are firstly extracted through the convolution layer, and the feature image with a size of $56 \times 56 \times 32$ is output. Then, a $1 \times 1 \times 32$ saliency graph is generated by summing up the activation values at the same position on the channel. The region of interest (ROI) is retrieved from the original CXR graph and mapped to the saliency graph. By increasing the weight of ROI, the linear model that can be explained and analyzed is more affected by $\mathrm{ROI}^{[15]}$. This improves the interpretation effect and makes the analysis results more reasonable. The attentional area effects are shown in Fig. 5. 

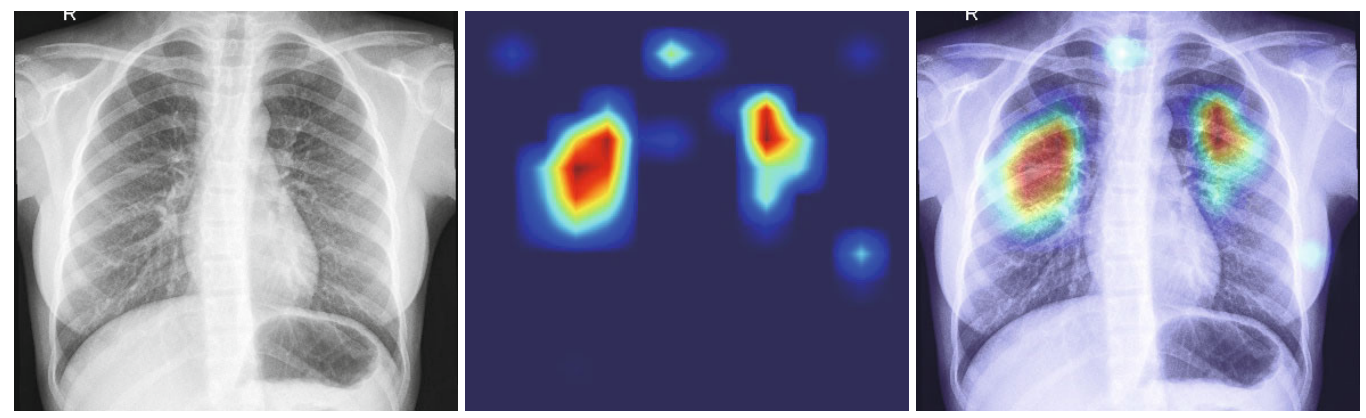

(a) Example 1
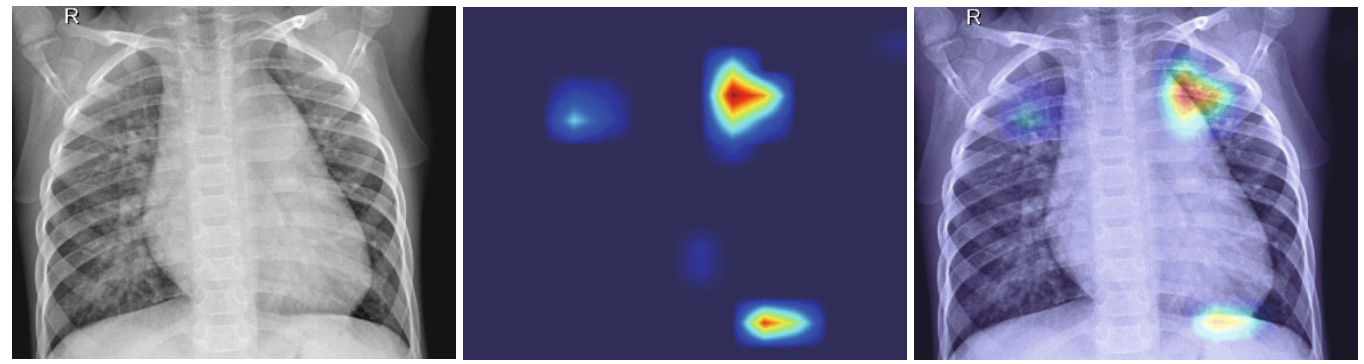

(b) Example 2

Fig. 5 Effect display of attention area

\section{Experimental Results and Analysis}

\subsection{Data Enhancement Operation}

Convolutional neural network has translation invariance, which makes it possible for data augmentation. Data augmentation is to make limited data produce more value without increase of other data. In the process of changing data dimension, the essence of data (hidden features) is unchanged, but the complexity of data (observable features) is enhanced to improve the robustness of mapping in complex environment.

Data can be augmented by rotating at specified an- gles, changing the size or other operations. In the code, the brightness enhancement function applies brightnessEnhancement (root_path, img_name), the contrast enhancement function applies contrastEnhancement (root_path, img_name), the image rotation function applies rotation (root_path, img_name), and the image flipping function applies flip (root_path, img_name). As shown in Fig. 6, the data are expanded 12 times by data enhancement operation, i.e., extending 25 pieces of COVID-19 CXRs to 300. Experiments show that the data augmentation method is feasible and has a positive impact on the judgment results.

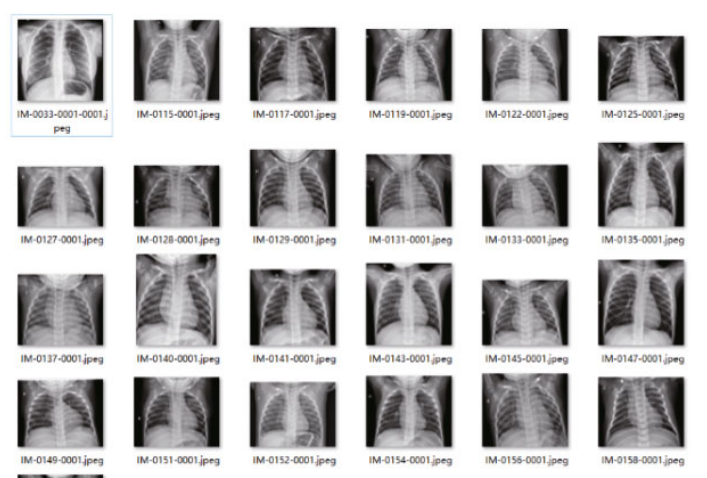

(a) Original

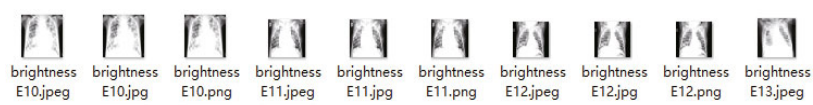

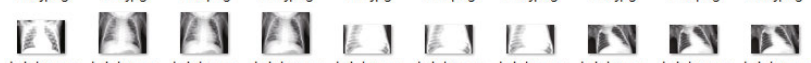
데

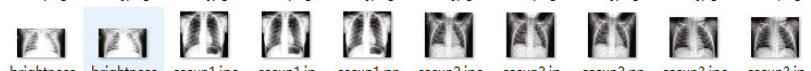

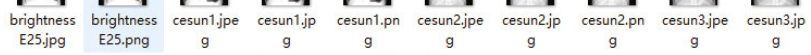

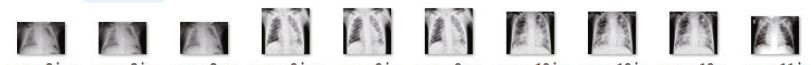
cesun8.jpe cesun8.jp cesun8.pn cesun9.jpe cesun9.jp cesun9.pn cesun10.jp cesun10.jp $\quad$ cesun10.p cesun11.jp

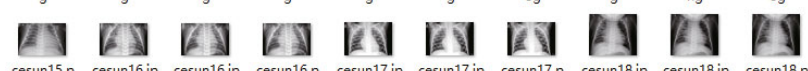

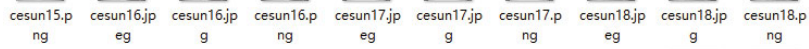

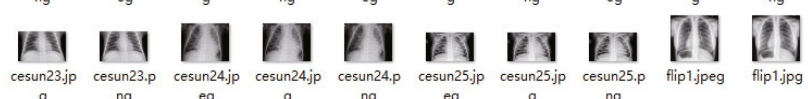

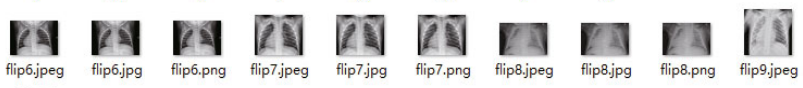

(b) Augmented

Fig. 6 CXR data 


\subsection{Experiment Comparison}

The design idea of the experiment is always emphasized in this paper, which is lightweight and interpretability. In this premise, we pursue the highest accuracy, recall rate and other parameters. Therefore, VGG-16, VGG-19, InceptionV3, and Xception were successively used as the backbones of experimental networks ${ }^{[16]}$. At the same time, three kinds of full connection layers designed with different structures have been tried with each backbone. Finally, each network was tested on the original dataset (OD, including 25 pieces of infected individuals' CXRs and 25 pieces of normal individuals' CXRs) and the augmented dataset (AD, including 300 pieces of infected individuals' CXRs and 300 pieces of normal individuals' CXRs).

Because we have to compare multiple groups of experiments, all the networks are trained on 1080ti. However, each network itself is lightweight enough to train easily on CPU-only devices. All networks train only 25 epochs and have similar accuracy to those trained with GPU equipment. Here, F1-score, a harmonic average of precision rate and recall rate, is considered. The experiment comparison results are shown in Table 2. For each network, the highest accuracy for the full connection structure, and the best global effect are highlighted in bold.

To achieve high precision CXR detection effect, we

Table 2 Results of different networks

\begin{tabular}{|c|c|c|c|c|c|c|}
\hline Dataset & Backbone & Dropout & $\mathrm{BN}$ & Accuracy & $\begin{array}{c}\text { Recall } \\
\text { rate }\end{array}$ & F1-score \\
\hline OD & VGG-16 & 0 & Yes & 0.8000 & 1.00 & 0.83 \\
\hline OD & VGG-16 & 0.5 & No & 0.9000 & 0.80 & 0.89 \\
\hline OD & VGG-16 & 0.5 & Yes & 0.9000 & 1.00 & 0.91 \\
\hline OD & VGG-19 & 0 & Yes & 0.8000 & 0.60 & 0.75 \\
\hline OD & VGG-19 & 0.5 & No & 0.7000 & 1.00 & 0.77 \\
\hline OD & VGG-19 & 0.5 & Yes & 0.9000 & 1.00 & 0.91 \\
\hline OD & InceptionV3 & 0 & Yes & 0.3000 & 0.20 & 0.22 \\
\hline OD & InceptionV3 & 0.5 & No & 0.5000 & 1.00 & 0.67 \\
\hline OD & InceptionV3 & 0.5 & Yes & 0.6000 & 0.80 & 0.67 \\
\hline OD & Xception & 0 & Yes & 0.7000 & 0.40 & 0.57 \\
\hline OD & Xception & 0.5 & No & 0.8000 & 0.80 & 0.80 \\
\hline OD & Xception & 0.5 & Yes & 0.9000 & 1.00 & 0.91 \\
\hline $\mathrm{AD}$ & VGG-16 & 0 & Yes & 0.9692 & 0.95 & 0.97 \\
\hline $\mathrm{AD}$ & VGG-16 & 0.5 & No & 0.9462 & 0.95 & 0.95 \\
\hline $\mathrm{AD}$ & VGG-16 & 0.5 & Yes & 0.9923 & 0.98 & 0.99 \\
\hline $\mathrm{AD}$ & VGG-19 & 0 & Yes & 0.8923 & 0.98 & 0.90 \\
\hline $\mathrm{AD}$ & VGG-19 & 0.5 & No & 0.9462 & 0.95 & 0.95 \\
\hline $\mathrm{AD}$ & VGG-19 & 0.5 & Yes & 0.9385 & 0.89 & 0.94 \\
\hline $\mathrm{AD}$ & InceptionV3 & 0 & Yes & 0.7538 & 0.68 & 0.73 \\
\hline $\mathrm{AD}$ & InceptionV3 & 0.5 & No & 0.7077 & 0.49 & 0.63 \\
\hline $\mathrm{AD}$ & InceptionV3 & 0.5 & Yes & 0.8615 & 0.88 & 0.86 \\
\hline $\mathrm{AD}$ & Xception & 0 & Yes & 0.8846 & 0.94 & 0.89 \\
\hline $\mathrm{AD}$ & Xception & 0.5 & No & 0.8154 & 0.86 & 0.82 \\
\hline $\mathrm{AD}$ & Xception & 0.5 & Yes & 0.9077 & 0.92 & 0.91 \\
\hline
\end{tabular}

tested complex deep networks including ResNet and SENet with attention mechanism. During the experiment, we repeatedly tried transfer learning, parameters adjusting, dropout, regularization and other operations, but the data demand caused by complex structure could not be met, and experimental results of the accuracy were all less than $70 \%$, so they were not listed in Table 2. According to the analysis of task requirements, the COVID-19 classification detection task is not complicated, and the classic CNN networks show efficient and accurate results in the case of few data. In the experiment, we used the control variable method to compare the three different structures of fully connected layers. Table 2 shows the four classical network structures with the highest precision and various experimental indicators.

After 25 epochs of training, the loss function curve and accuracy curve are shown in Fig. 7. From the curve trend, we can conclude that the effect of the model is satisfactory.

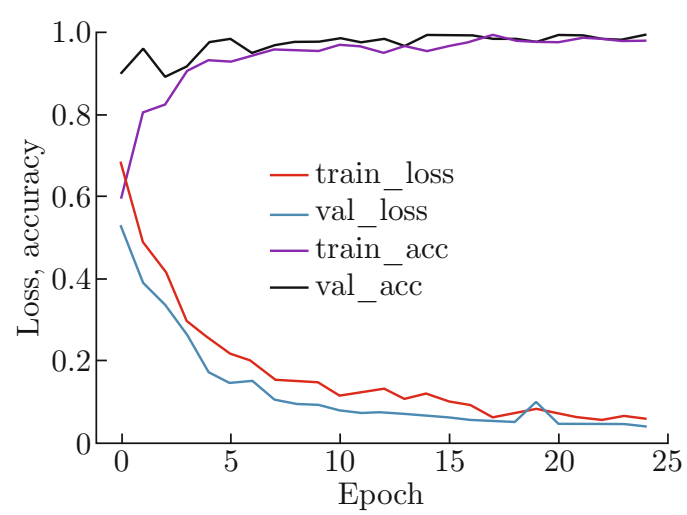

Fig. 7 Loss function and accuracy curves on COVID-19 dataset

\subsection{Interpretability Analysis}

There are some parameters that need to be specified when using the "Lime" method for interpretability analysis. The "positive_only" parameter indicates whether only areas that have a positive effect on discriminant discrimination are marked. This value has been chosen to be "False" because we want to provide a more comprehensive auxiliary analysis for medical workers by comparing the representative regions of the normal CXR with those of infected individual's CXR. The "num_features" parameter indicates the number of the regions with the largest contributions. In addition, "hide_rest = False" means that the rest of the CXR pixel blocks are displayed together after the suspicious area has been marked. If the value is true, the rest of the CXR will be painted gray. Then, the "for loop" iterates over the dataset and the explanation of each image is displayed.

The interpretability analysis effect of CXR is shown in Fig. 8. The left image is a CXR that is judged to 
be normal. As seen from the angle of CXR, this is a sample augmented with data augmentation techniques. The green areas are the areas that make a positive contribution to the model's judgment of normal individual. The right one is a CXR of a person who has been identified as infected. This is a CXR sample from the original dataset, and the areas in red are the areas that make a positive contribution to the model's judgment whether it is infected.

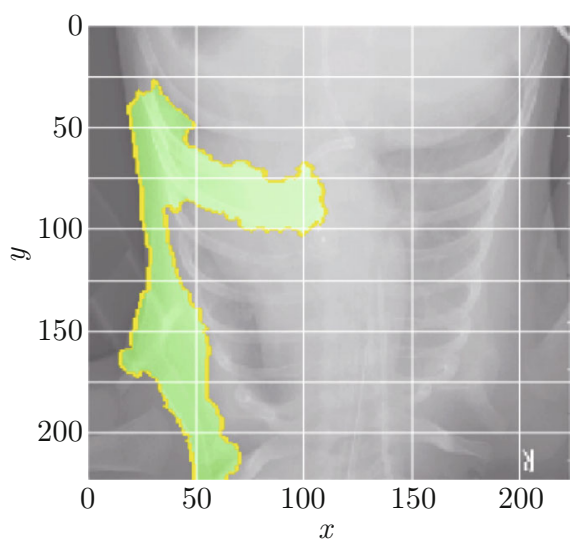

(a) Normal individual
In Fig. 8, the distribution of interpreted regions in CXR after interpretability analysis is not limited to lungs but distributed throughout the thoracic cavity, which may mislead medical diagnosis and pathological analysis. By adding attention weight to the interpretability analysis, the model more focuses on the lung area in the center of CXR. The interpretability analysis results generated by the improved "Lime" of attention weight are shown in Fig. 9.

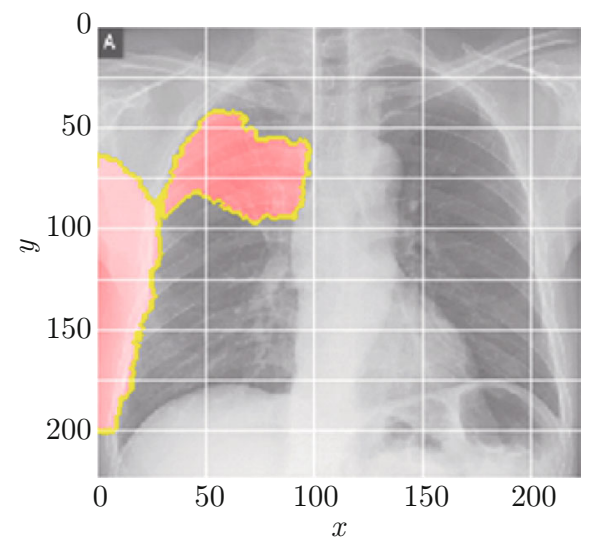

(b) Infected individual

Fig. 8 Interpretation of normal and infected individual's CXR

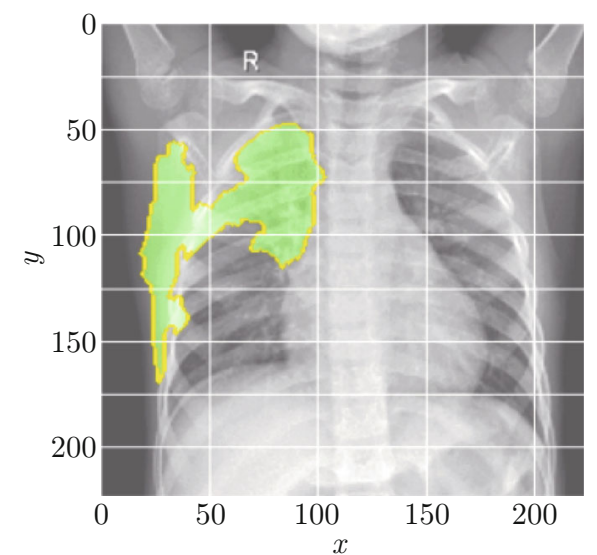

(a) Example 1 of normal individual

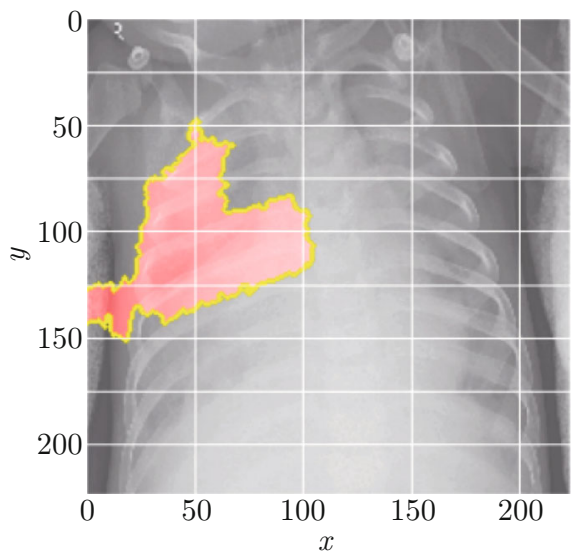

(c) Example 1 of infected individual

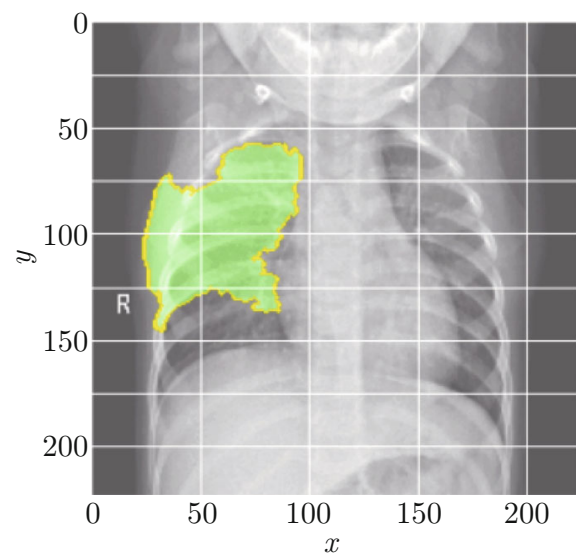

(b) Example 2 of normal individual

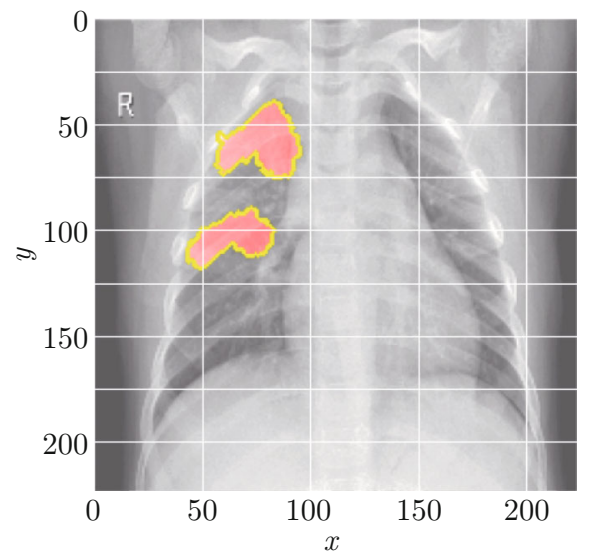

(d) Example 2 of infected individual

Fig. 9 Improved CXR interpretation effect of normal and infected individuals 


\section{Conclusion}

The researches on COVID-19 still need to be sustained. Nowadays, AI technology and network structure are updated rapidly. It is more important to choose a suitable model, which will result in a multiplier effect. The difficulty of insufficient data in an emergency is a warning for AI researchers. To solve the practical difficulties, on the one hand, the lightweight VGG-16 network and the pre-trained model were used to train the improved full connection layer only through the precious COVID-19 sample. On the other hand, the dataset was expanded by means of data augmentation. Satisfactory results were obtained by combining the two methods.

It is different from machine learning that the applications of deep learning are often not interpretable. It is more like "black box" when dealing with problems. Only input and output can be observed, which means that we do not understand the internal principles of the model. This question is often overlooked because of the high accuracy of AI models. However, it is a combination of risk and opportunity. We want the model to explain the criterion for judgment, and then we can analyze the "mindset" of the model. AI and our human beings are qualitatively different. Perhaps, the way of thinking of machines can provide a boost for human progress. At least it would not make individuals anxious because it is not interpretable.

The purpose of scientific research is always to benefit more individuals, which is also the reason why we give up CT data and choose CXR. Because of the high penetration rate and low cost of CXR, the COVID-19 detection system based on CXR is of greater value and can help more individuals. Meanwhile, CXR diagnosis can not only contribute to the health and epidemic prevention work, but also find more potential data, thus forming a positive cycle to solve COVID-19 as soon as possible, and making a contribution to the fight against the COVID-19.

\section{References}

[1] CHEN N S, ZHOU M, DONG X, et al. Epidemiological and clinical characteristics of 99 cases of 2019 novel coronavirus pneumonia in Wuhan, China: A descriptive study [J]. The Lancet, 2020, 395(10223): 507-513.

[2] LU Y Q, SHEN L, HE B. Application of artificial intelligence in assisted diagnosis and treatment of cardiovascular disease [J]. Journal of Shanghai Jiaotong University (Medical Science), 2020, 40(2): 259-262 (in Chinese).

[3] ZHANG L, CHEN Q, JIANG B B, et al. Preliminary study on motion artifacts removal of coronary CT angiography using generative adversarial network [J]. Journal of Shanghai Jiao Tong University (Medical Science), 2020, 40(9): 1229-1235 (in Chinese).
[4] LIU Y H, ZHANG F D, ZHANG Q Y, et al. Cross-view correspondence reasoning based on bipartite graph convolutional network for mammogram mass detection [C]//2020 IEEE/CVF Conference on Computer Vision and Pattern Recognition (CVPR). Seattle, WA: IEEE, 2020: 3811-3821.

[5] RIBEIRO M T, SINGH S, GUESTRIN C. "Why should I trust you?" Explaining the predictions of any classifier $[\mathrm{C}] / /$ Proceedings of the 22nd ACM SIGKDD International Conference on Knowledge Discovery and Data Mining. San Francisco, CA: ACM, 2016: 11351144.

[6] PALATNIK DE SOUSA I, VELLASCO M M B R, COSTA DA SILVA E. Local interpretable modelagnostic explanations for classification of lymph node metastases [J]. Sensors, 2019, 19(13): 2969.

[7] SIMONYAN K, ZISSERMAN A. Very deep convolutional networks for large-scale image recognition [EB/OL]. [2021-04-12]. https://arxiv.org/abs/1409.1556.

[8] SZEGEDY C, VANHOUCKE V, IOFFE S, et al. Rethinking the inception architecture for computer vision [EB/OL]. [2021-04-12]. https://arxiv.org/abs/ 1512.00567

[9] HU X C, MU H Y, ZHANG X Y, et al. Meta-SR: A magnification-arbitrary network for super-resolution [C]//2019 IEEE/CVF Conference on Computer Vision and Pattern Recognition (CVPR). Long Beach, CA: IEEE, 2019: 1575-1584.

[10] ZHANG X F, WU G. Data augmentation method based on generative adversarial network [J]. Computer Systems Applications, 2019, 28(10): 201-206 (in Chinese).

[11] BRUNESE L, MERCALDO F, REGINELLI A, et al. Explainable deep learning for pulmonary disease and coronavirus COVID-19 detection from X-rays $[\mathrm{J}]$. Computer Methods and Programs in Biomedicine, 2020, 196: 105608 .

[12] COZZI D, ALBANESI M, CAVIGLI E, et al. Chest Xray in new Coronavirus Disease 2019 (COVID-19) infection: Findings and correlation with clinical outcome [J]. La Radiologia Medica, 2020, 125(8): 730-737.

[13] SCHIAFFINO S, TRITELLA S, COZZI A, et al. Diagnostic performance of chest X-ray for COVID-19 pneumonia during the SARS-CoV-2 pandemic in Lombardy, Italy [J]. Journal of Thoracic Imaging, 2020, 35(4): W105-W106.

[14] CHENG K Y, SHI W X, ZHAN Y Z. Research Progress on explicability of deep learning [J]. Journal of Computer Research and Development, 2020, 57(6): 12081217 (in Chinese).

[15] SELVARAJU R R, COGSWELL M, DAS A, et al. Grad-CAM: Visual explanations from deep networks via gradient-based localization [J]. International Journal of Computer Vision, 2020, 128(2): 336-359.

[16] ANTHIMOPOULOS M, CHRISTODOULIDIS S, EBNER L, et al. Lung pattern classification for interstitial lung diseases using a deep convolutional neural network [J]. IEEE Transactions on Medical Imaging, 2016, 35(5): 1207-1216. 